\title{
Turbulence: a new zero-equation model
}

\author{
K. Alammar \\ Department of Mechanical Engineering King Saud University, \\ Saudi Arabia
}

\begin{abstract}
A new, zero-equation turbulence model for average turbulent fluid flow was developed. The new model, which is based on the Boussinesq hypothesis, incorporates the wall effect and predicts behaviour of the effect with increasing distance from the wall. It is applied to incompressible two-dimensional and axisymmetric turbulent flows over a flat plate and in pipes, respectively. In the fully turbulent regions, predictions agree well with published measurements for skin friction coefficient in the range of Reynolds numbers considered, up to 10 million for both the flat plate and pipe without adjustments to the model. No wall functions were implemented. For a given roughness, the skin friction for the pipe was shown to be independent of the Reynolds number in two test cases, namely Re 1 and 10 million. The skin friction and velocity profiles are presented and assessed.
\end{abstract}

Keywords: turbulence modelling, wall effect, flat plate, pipe, skin friction.

\section{Introduction}

The problem of turbulence dates back to the days of Claude-Louis Navier and George Gabriel Stokes, as well as others in the early nineteenth century. Searching for its solution, it was a source of great despair for many notably great scientists, including Werner Heisenberg, Horace Lamb, and many others. The complete description of turbulence remains one of the unsolved problems in modern physics.

The history of turbulence spans a period of nearly two centuries, and one can spend considerable time and effort to compile its lengthy history and the many efforts that have been put to resolve it. However, this paper is not intended the least to present history of turbulence. Instead, objective of the present work is to present a zero-equation model that may pave the way to the solution to average 
turbulent flows. For validation, incompressible flows over smooth flat plate and pipe are considered.

\section{Theory}

Starting with the incompressible Navier-Stokes' in Cartesian index notation, and with Reynolds decomposition, averaging, and following Boussinesq hypothesis [1], we have:

$$
\begin{gathered}
\frac{\partial\left(\bar{u}_{i}\right)}{\partial x_{i}}=0 \\
\rho\left[\frac{\partial\left(\bar{u}_{i}\right)}{\partial t}+\left(\bar{u}_{j}\right) \frac{\partial\left(\bar{u}_{i}\right)}{\partial x_{j}}\right]+\frac{\partial(\bar{p})}{\partial x_{i}}-\frac{\partial}{\partial x_{j}} \mu\left(\frac{\partial \bar{u}_{i}}{\partial x_{j}}+\frac{\partial \bar{u}_{j}}{\partial x_{i}}\right)= \\
\frac{\partial}{\partial x_{j}} \mu_{t}\left(\frac{\partial \bar{u}_{i}}{\partial x_{j}}+\frac{\partial \bar{u}_{j}}{\partial x_{i}}\right)
\end{gathered}
$$

For simplicity, the normal stresses (except for the thermodynamic pressure) and body forces are neglected. $\mu_{t}=C \operatorname{Re}_{w} \mu$ is the dynamic eddy viscosity. $\mathrm{C}$ is a non-dimensional function of the wall roughness. For a smooth wall, it is a constant. For isotropic roughness, it is a different constant. $\operatorname{Re}_{w}=u_{i} \rho d / \mu$, and $\mathrm{d}$ is the normal distance from the wall. If eq. (2) is normalized, the shear stresses result in the following:

$$
\frac{\partial}{\partial x_{j}}\left[\mathrm{Re}^{-1}+C \frac{u_{i} d}{U L}\right]\left(\frac{\partial \bar{u}_{i}}{\partial x_{j}}+\frac{\partial \bar{u}_{j}}{\partial x_{i}}\right)
$$

The second term in the brackets is a non-dimensional number attributed to the wall. Clearly, it dominates at high Reynolds number flows. In absence of walls, however, a controversy arises because turbulence is known to exit even in absence of wall effects. In this case, one plausible length scale would be the mean free path, instead of the distance from the wall. This would give rise to second-order effects that would be negligible in presence of walls, and therefore should not affect our results in the present study.

\section{The numerical procedure}

Assuming two-dimensional and axisymmetric flows for the flat plate and pipe, respectively, equations (1) and (2) were solved using SIMPLE [2], and secondorder schemes. The constant $\mathrm{C}$ was set to 0.016 for both cases. Gauss-Seidel iterative method was used on a 32-bit laptop using 100,000 structured grids. 


\section{Results and discussion}

The mean velocity and skin friction coefficient for the flat plate and pipe are depicted in fig. 1. The data is presented on a log scale. For the flat plate, the Reynolds number extends from zero (leading edge) to $1.0 \times 10^{7}$. The agreement with measurements of Wieghardt and Tillman [3] is good starting from roughly Re $5 \times 10^{5}$, i.e., after transition. Laminar effects are expected to lower the skin friction within the transitional region. For the pipe flow, Reynolds number extends from 10,000 to $1.0 \times 10^{7}$. Again, good agreement is attained with measurements of Moody [4], except within the transitional region.

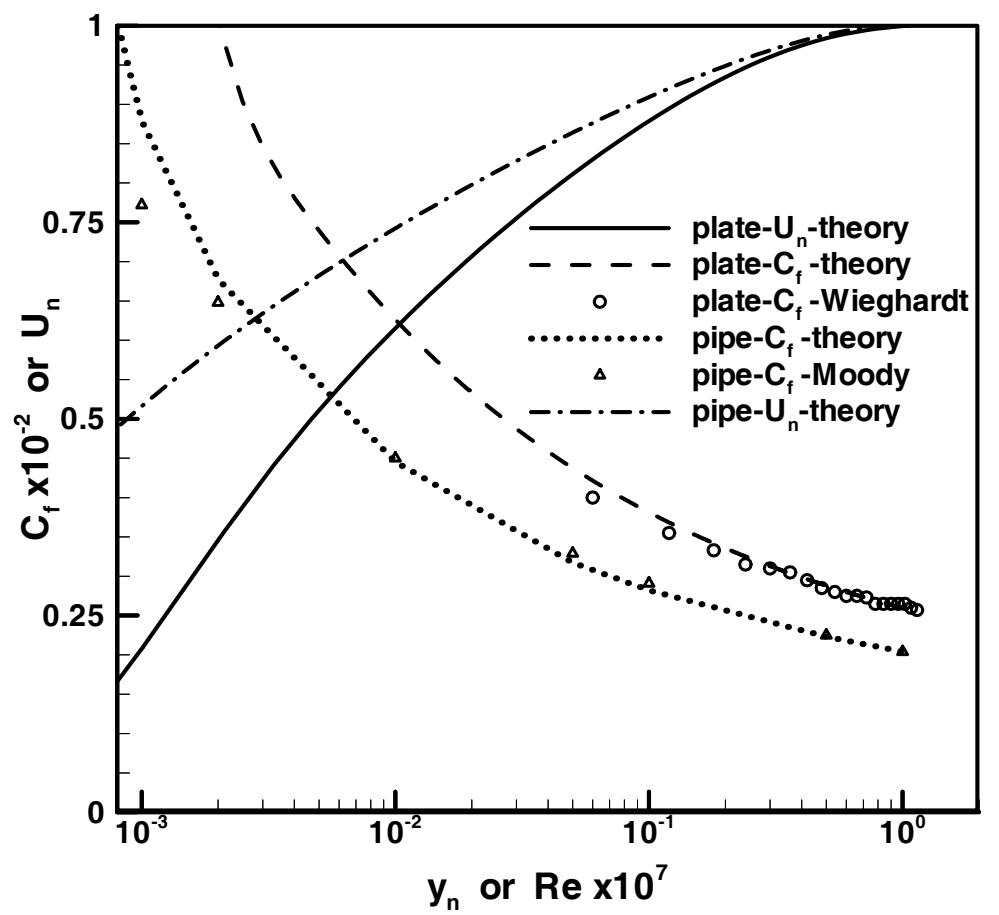

Figure 1: $\quad$ Skin friction coefficient and velocity profiles.

The mean velocity profiles are presented for Re $5.0 \times 10^{6}$ for both cases. Clearly, the boundary layer is considerably thinner for the pipe. Since pipe walls surround the flow, turbulence production is greater in the case of pipe flow. This leads to more energizing of the boundary layer, and hence thinner boundary layer. $\mathrm{y}^{+}$in this case was in the order of 0.001 for the pipe and 1.0 for the plate.

Though not shown in fig. 1, effect of the wall roughness was demonstrated with a test case for which $\mathrm{C}$ was set to 0.7 . This value was chosen to reproduce a skin friction coefficient value of 0.0026 at $\operatorname{Re} 1.0 \times 10^{7}$, which corresponds to a relative roughness parameter of 0.003 in the Moody chart. Using the same value 
for $\mathrm{C}$, no significant change in the skin friction coefficient was predicted at $\mathrm{Re}$ $1.0 \times 10^{6}$, hence predicting the independence of skin friction on Reynolds number at certain roughness levels.

\section{Conclusions}

Steady, incompressible, two-dimensional turbulent flow over a flat plate and in a pipe was simulated using a new zero-equation turbulence model. The model predicts behaviour of the wall effect with increasing distance from the wall. The theoretical results agreed well with published measurements for both the flat plate and pipe in the fully turbulent regions. The developed model is applicable to fluid flow in general. Other transport equations, including the energy and species, are solved accordingly.

\section{Nomenclature}

C a non-dimensional function of wall roughness

$\mathrm{C}_{\mathrm{f}}=\tau_{w} / 0.5 \rho U^{2}$

$\mathrm{D}$ pipe diameter, $\mathrm{m}$

$\mathrm{d}$ normal distance from the wall, $\mathrm{m}$

$\mathrm{L} \quad$ length scale, $\mathrm{m}$. D for the pipe and $\mathrm{x}_{1}$ for the plate.

$\operatorname{Re} \quad=U \rho L / \mu$

$\operatorname{Re}_{\mathrm{w}}=u_{i} \rho d / \mu$

$\mathrm{U} \quad$ velocity scale, $\mathrm{m} / \mathrm{s}$. Centreline for the pipe and freestream for the plate

$\mathrm{U}_{\mathrm{n}} \quad$ velocity normalized by the centreline for the pipe and freestream for the plate.

$\mathrm{u}_{\mathrm{i}} \quad$ velocity component, $\mathrm{m} / \mathrm{s}$

$\mathrm{x}_{\mathrm{i}} \quad$ Cartesian coordinate, $\mathrm{m}$

$\mathrm{y}^{+} \quad$ non-dimensional wall distance

$\mu \quad$ fluid dynamic viscosity, $\mathrm{Pa} \mathrm{s}$

$\rho \quad$ fluid density, $\mathrm{kg} / \mathrm{m}^{3}$

$\tau_{w} \quad$ wall shear stress, $\mathrm{Pa}$.

\section{References}

[1] Schlishting, H. \& Gersten, K., Boundary layer Theory, Springer, Berlin, 2000.

[2] Patankar, S.V. \& Spalding, D.B., A calculation procedure for heat, mass and momentum transfer in three-dimensional parabolic flows, International Journal of Heat and Mass Transfer, 15, pp. 1787-1806, 1972.

[3] Wieghardt, K. \& Tillman, W., On the Turbulent Friction Layer for Rising Pressure, NACA TM-1314, 1951.

[4] Moody, L.F., Friction Factors for Pipe Flow, Transactions of the A.S.M.E., pp. 671-684, 1944. 